\title{
Activity-Dependent pH Shifts and Periodic Recurrence of Spontaneous Interictal Spikes in a Model of Focal Epileptogenesis
}

\author{
Marco de Curtis, ${ }^{1}$ Alfredo Manfridi, ${ }^{2}$ and Gerardo Biella ${ }^{1}$ \\ 1Department of Experimental Neurophysiology, Istituto Nazionale Neurologico, 20133 Milan, Italy and 2Institute of Human \\ Physiology II, University of Milan, 20133 Milan, Italy
}

\begin{abstract}
The mechanisms that control the periodicity of spontaneous epileptiform cortical potentials were investigated in the in vitro isolated guinea pig brain preparation. A brief intracortical application of bicuculline in the piriform cortex induced spontaneous interictal spikes (s/Ss) that recurred with high periodicity $(8.5 \pm 3.1 \mathrm{sec}$, mean $\pm \mathrm{SD})$. Intracellular recordings from principal neurons showed that the early phase of the inter-s/S period is caused by a GABAb receptor-mediated inhibitory potential. The late component of the interspike period correlated to a slowly decaying depolarization abolished at membrane potentials positive to $-32.1 \pm 5.3 \mathrm{mV}$ and was not associated with membrane conductance changes. Specific pharmacological tests excluded the contribution of synaptic and intrinsic conductances to the late inter-s/S interval. Recordings with ion-sensitive electrodes demonstrated that $s / S s$ determined both a rapid increase in extracellular $\mathrm{K}^{+}$concentration (0.5-1 $\mathrm{mm})$ and an extracellular alkalinization $(0.05-0.08 \mathrm{pH}$ units) that slowly decayed during the inter-s/S period and returned to control values just before a subsequent $s / S$ was
\end{abstract}

generated. These observations were not congruous with the presence of a silent period, because both extracellular increase in $\mathrm{K}^{+}$and alkalinization are commonly associated with an increase in neuronal excitability. Extracellular alkalinization could be correlated to an s/S-induced intracellular acidification, a phenomenon that reduces cell coupling by impairing gap junction function. When intracellular acidification was transiently prevented by arterial perfusion with $\mathrm{NH}_{4} \mathrm{Cl}(10-20 \mathrm{mM})$, spontaneous ictal-like epileptiform discharges were induced. In addition, the gap junction blockers octanol (0.2-2 mM) and 18 - $\alpha$-glycyrrethinic acid $(20 \mu \mathrm{M})$ applied either via the arterial system or locally in the cortex completely and reversibly abolished the $s / S$. The results reported here suggest that the massive cell discharge associated with an $s / S$ induce a strong inhibition, possibly secondary to a $\mathrm{pH}$-dependent uncoupling of gap junctions, that regulates $s / S$ periodicity.

Key words: epileptogenesis; interictal spikes; isolated brain preparation; periodic activity; $\mathrm{pH}$; piriform cortex
When a condition of hyperexcitability occurs in the cortex, epileptiform events such as interictal spikes or ictal discharges arise spontaneously. In vivo and in vitro studies that used different acute and chronic models of epileptogenesis showed that spontaneous interictal spikes $(s I S \mathrm{~s})$ can recur with a period variable between 1 and $10 \mathrm{sec}$ depending on the experimental condition (Prince 1971; Lebovitz, 1979; Traub and Wong, 1982; Rutecki et al., 1985; Schneiderman and Mac Donald 1989; Chamberlin et al., 1990; Leung, 1990; Perez-Velasquez et al., 1994; Pelletier and Carlen, 1997). Clinical observations demonstrated that periodic spiking activity is a common phenomenon in lesional human epilepsy (Chatrian et al., 1964) and in idiopathic benign partial epilepsies of childhood (Beaussat et al., 1972). According to the work of Lebovitz (1979), autorhythmicity of $s I S$ s derives from the functional suppression of the propagation to the soma of the spontaneous synaptic events generated distally in the dendrites. This observation introduced the idea that $s I S$ periodicity is not simply attributable to progressive buildup of excitation but might be caused by a prolonged and powerful phasic inhibition that follows

\footnotetext{
Received April 13, 1998; revised June 30, 1998; accepted June 30, 1998.

Partial support was provided by the Italian Health Ministry through PharmaciaUpjohn, Italy (PNR-MURST Grant 1.3.3.3). G.B. is supported by a Human Frontier Science Program fellowship (Grant RG 19/96). We thank Dario Brambilla and Carlo Rossetti for the technical help with the preparation of the ion-selective recordings.

Correspondence should be addressed to Marco de Curtis, Department of Experimental Neurophysiology, Istituto Nazionale Neurologico, via Celoria 11, 20133 Milan, Italy.

Copyright (C) 1998 Society for Neuroscience $\quad 0270-6474 / 98 / 187543-09 \$ 05.00 / 0$
}

the synchronous paroxysmal discharge associated with the sIS itself. This assumption will be tested here in an acute model of focal epileptogenesis induced in the piriform cortex of the in vitro isolated guinea pig brain preparation. Previous studies on this model demonstrated that a transient ejection of bicuculline in the piriform cortex induce $s I S$ s that recur periodically and persist for hours, even when the drug is washed out (de Curtis et al., 1994, 1998; Forti et al., 1997). The $s I S$ s are sustained by a primary burst associated with the activation of an intrinsic calcium spike, followed by a large recurrent glutamatergic synaptic potential propagated along the diffuse intrinsic associative fiber system of the piriform cortex (Haberly and Bower, 1989; Biella and de Curtis, 1995). The intracellular and extracellular events associated with the interspike silent period will be analyzed in the present study.

\section{MATERIALS AND METHODS}

Experiments were performed on young adult guinea pigs (200-250 gm; Charles River, Calco, Italy). The procedures for the isolation of the brain have been previously described in detail (Llinas et al., 1981; de Curtis et al., 1991; Muhlethaler et al., 1993). Briefly, after barbiturate anesthesia (Pentotal, $20 \mathrm{mg} / \mathrm{kg}$, i.p.) and after cardiac perfusion with cold saline solution, the brain was extracted and transferred to an incubation chamber. The in vitro brain was perfused with an oxygenated solution $(5 \%$ $\mathrm{CO}_{2}-95 \% \mathrm{O}_{2}$ ) via a cannula inserted into the basilar artery. The composition of the perfusate was: $\mathrm{NaCl} 126 \mathrm{~mm}, \mathrm{KCl} 2.3 \mathrm{~mm}, \mathrm{NaHCO}_{3} 26$ $\mathrm{mM}, \mathrm{MgSO}_{4} 1.3 \mathrm{~mm}, \mathrm{CaCl}_{2} 2.4 \mathrm{~mm}, \mathrm{KH}_{2} \mathrm{PO}_{4} 1.2 \mathrm{~mm}$, glucose $15 \mathrm{~mm}$, HEPES $5 \mathrm{~mm}$, thiourea $0.4 \mathrm{~mm}$, and $3 \%$ dextran $70.000, \mathrm{pH} 7.3$. The perfusion rate was $5.5-6 \mathrm{ml} / \mathrm{min}$. The experiments were performed at 


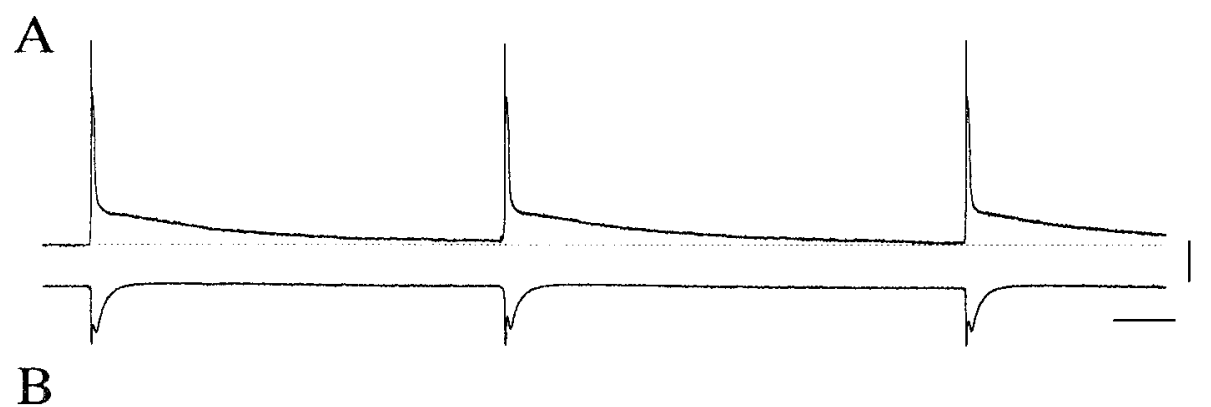

Figure 1. Periodic spontaneous interictal spikes $(s I S s)$ recorded intracellularly (top traces) and extracellularly (bottom traces) from the anterior piriform cortex $\sim 2 \mathrm{hr}$ after local intracortical injection of bicuculline. $A$, Three consecutive $s I S \mathrm{~s}$ are shown. Each $s I S$ is followed by a slow depolarization that returns to control level just before the next $s I S$ is generated. $B$, When the membrane potential was artificially depolarized by a steady intracellular current injection, a slow afterhyperpolarizing potential (arrow) was unmasked during the early component of the inter-sIS period; such a potential has been previously demonstrated to be mediated by GABAb receptors (de Curtis et al., 1998). Resting membrane potentials (dotted lines) were -81 and $-78 \mathrm{mV}(A, B$, respectively).
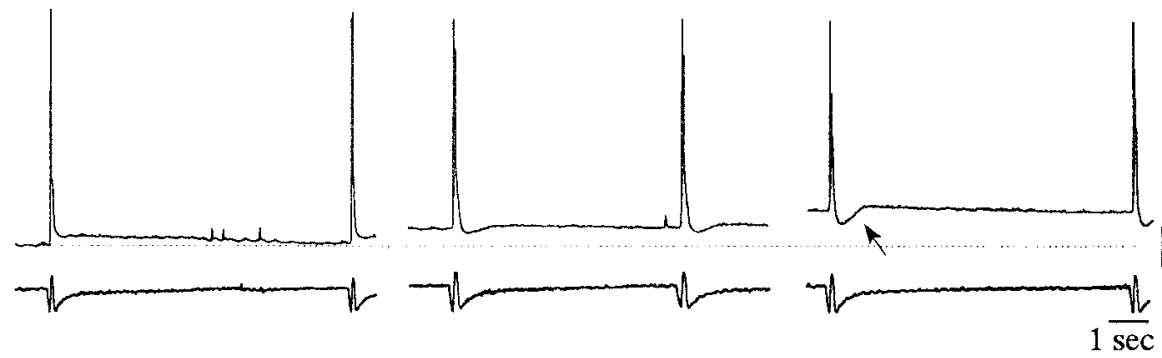

$20 \mathrm{mV}$ $1 \mathrm{mV}$ $32^{\circ} \mathrm{C}$. Bicuculline methiodide $(2 \mathrm{~mm}$; Research Biochemicals, Natick, MA) dissolved in the perfusate was injected for $10 \mathrm{sec}$ at $800 \mu \mathrm{m}$ depth in the anterior piriform cortex (APC) through a 8- to 10- $\mu$ m-tip diameter glass pipette connected to a graduated syringe. One hundred fifty to 200 nmol of bicuculline were delivered in one single ejection; the pipette was removed after bicuculline application. Bicuculline was washed out within 90 min (de Curtis et al., 1994; Forti et al., 1997). The experimental protocol has been reviewed and approved by the Commitee on Animal Care and Use of the Istituto Nazionale Neurologico.

Although the study is centered on spontaneous events, a bipolar silver wire electrode was positioned on the lateral olfactory tract (LOT) to evoke responses in the APC and to locate the depth of the extracellular recording electrodes (3-10 $\mathrm{M} \Omega$ resistance micropipettes filled with $0.9 \%$ $\mathrm{NaCl}$ ). Intracellular recordings were performed from principal neurons in layers II and III with sharp electrodes filled either with $3 \mathrm{~m} \mathrm{~K}$-acetate or with $2 \mathrm{M} \mathrm{K}$-acetate and $1 \%$ biocytin (50-120 M $\Omega$ resistance). Extracellular and intracellular activity was recorded with a Neurodata (New York, NY) amplifier. Extracellular $\mathrm{K}^{+}$concentration and $\mathrm{pH}$ were measured with ion-sensitive microelectrodes (ISMs) pulled from borosilcate glass capillaries with filament (WPI; electrode tip diameter, 2-4 $\mu \mathrm{m})$ acid-cleaned and dryed at $100^{\circ} \mathrm{C}$. The pipettes were then exposed for 1 min to dimethyldichlorosylane vapors (14896; Fluka, Neu-Ulm, Germany) and baked at $120^{\circ} \mathrm{C}$ for $2 \mathrm{hr}$. The $\mathrm{K}^{+}$-ISMs were filled at the tip with potassium ionophore I-cocktail A (Fluka 60031) and were backfilled with $200 \mathrm{~mm} \mathrm{KCl}$. The pH-ISM tips were filled with hydrogen ionophore II-cocktail A (Fluka 95297) and back-filled with a buffer solution (in mM: $\mathrm{NaCl} 100$, HEPES, and $\mathrm{NaOH} 10, \mathrm{pH} 7.5$ ). $\mathrm{K}^{+}$and $\mathrm{pH}$ calibration solutions were similar to the perfusate used during the experiment, with either $\mathrm{KCl}$ or $\mathrm{NaHCO}_{3}$ substituted for the corresponding moles of $\mathrm{NaCl}$; for the $\mathrm{K}^{+}$-ISM the perfusate solution was modified to contain $1-20 \mathrm{~mm} \mathrm{~K}^{+}$. For the $\mathrm{pH}$-ISM the perfusate solution was modified from $\mathrm{pH} 6.0$ to 8.0 ( $\mathrm{pH}$ range measured with the conventional macro-pH electrode calibrated with commercial buffers). The signals were amplified with a high input impedance head stage and an Axoclamp 2B amplifier (Axon Instruments). The $\mathrm{pH}-\mathrm{ISMs}$ had a response of 50-55 $\mathrm{mV}$ for unit change in $\mathrm{pH}$. The data were accepted only if the calibration curves obtained before and after the experiments did not differ by $>5 \%$. Signals were stored on a Biological 2602 digital tape recorder for off-line analysis with a Digital Microvax 3400 computer system.

Octanol (0.2 mM; Sigma, St. Louis, MO)(S)-34-methyl-4-carboxyphenylglycine (MCPG, 2 mM; Tocris), 18- $\alpha$-glycyrrethinic acid (Sigma, $20 \mu \mathrm{M})$, and $\mathrm{NH}_{4} \mathrm{Cl}(10-20 \mathrm{~mm}$, substituted for $\mathrm{NaCl}$; Sigma) were delivered by arterial perfusion. When octanol and MCPG were applied locally in the extracellular space, a 10-fold concentration was used. QX-314 (80 mM; Research Biochemicals) dissolved in $1 \mathrm{~m} \mathrm{~K}$-acetate, $\mathrm{pH}$ 7.3, was applied by intracellular diffusion through the recording pipette. When micropipettes filled with biocytin were used for intracellular staining, the isolated brains were fixed overnight with $4 \%$ paraformaldehyde. Coronal sections (100 $\mu \mathrm{m}$ thick) were then processed for avidin-
HRP visualization and were counterstained with neutral red to locate the cells within the cortical layers (Forti et al., 1997).

\section{RESULTS}

The silent inter-sIS period was analyzed by performing simultaneous extracellular field recordings and intracellular recordings from principal neurons in layers II and III of the APC at the bicuculline focus. Average resting membrane potential was $-75.20 \pm 6.47 \mathrm{mV}$ (mean $\pm \mathrm{SD})$. No differences in the electrophysiological behavior during the inter-sIS period were observed between the layer II $(n=29)$ and layer III $(n=9)$ cells.

As previously reported (de Curtis et al., 1998), a single injection of bicuculline in the deep layers of the APC induces $s I S$ s that recur periodically every $8.5 \pm 3.1 \mathrm{sec}$ (Fig. $1 A$ ) and persist when bicuculline is washed out. The frequency of the $s I S \mathrm{~s}$ became regular in an individual brain $10 \mathrm{~min}$ after the application of bicuculline. Previous studies demonstrated that the extracellularly recorded $S I S$ correlates to a primary burst of action potentials subtended by a calcium spike, followed by a secondary depolarization mediated by a recurrent glutamatergic excitatory potential (Forti et al., 1997; de Curtis et al., 1998; also see Traub and Wong, 1982; Traub et al., 1993). During the silent inter-sISs period a slowly decaying depolarization was recorded intracellularly. The activation of the next sIS occurred exclusively when the membrane potential returned to resting values (Fig. 1A). As previously demonstrated, the early portion of the inter-s $I S$ period is attributable to a $\mathrm{K}^{+}$-dependent $\mathrm{GABAb}$ receptor-mediated inhibitory potential (de Curtis et al., 1998). The GABAb inhibitory potential was identified as a hyperpolarizing afterpotential when the membrane was depolarized to values more positive then $-75 \mathrm{mV}$ by injecting intracellularly a steady current (Fig. $1 \mathrm{~B}$, right trace, arrow). The modifications of membrane potential induced by current injection did not change the frequency of $s I S$ repetition. The late, slowly decaying component of the inter-sIS potential showed no voltage reversal but was abolished at membrane potentials positive to $-32.1 \pm 5.3 \mathrm{mV}(n=13)$. The late inter$s I S$ s period can be imputed either to the activation of an inhibitory membrane conductance or to a passive decrease in excitability associated with microenvironmental changes in the extracellular space. 

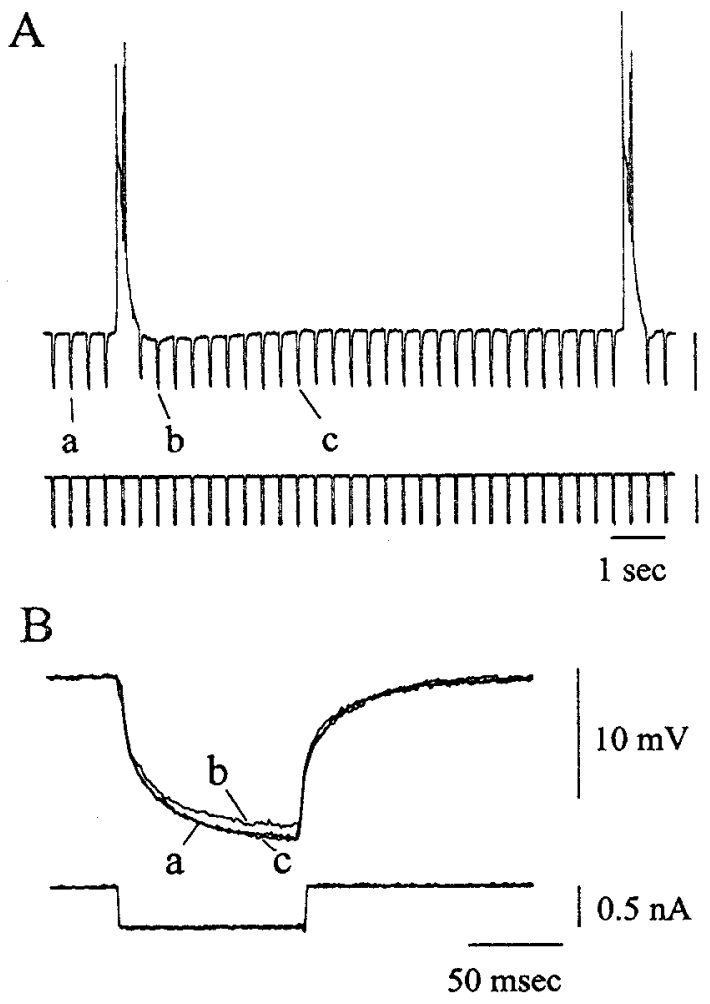

Figure 2. The late part of the inter-sIS period is not associated with an increase in membrane conductance. A $100 \mathrm{msec}$ hyperpolarizing current pulse (monitored in the bottom traces in each panel) applied at $3 \mathrm{~Hz}$ was used to test changes in membrane resistance during the interval between two $s I S$ s. The membrane potential was depolarized $(-55 \mathrm{mV}$ from a resting membrane potential of $-77 \mathrm{mV}$ ) to enhance the postburst afterhyperpolarization. The voltage responses indicated in $A$ are reported in $B$ at higher time resolution. The membrane resistance decreased just after the $s I S(b)$ and returned to control pre-sIS values within 2 sec (compare traces $a, c$, recorded before and 3 after the $s I S)$.

To verify whether the late inter-sIS potential correlated to the activation of a membrane conductance, cellular membrane resistance was tested continuously during several consecutive $s I S$ s by evaluating the voltage response to a 50-100 msec hyperpolarizing current pulse at 2-3 Hz. As illustrated in Figure 2, membrane resistance decreased just after the $s I S$, returned to pre-sIS values within $2 \mathrm{sec}$, and was not altered during the late part of the inter-s $I S$ potential $(n=8)$. In Figure $2 B$ the voltage responses to the current pulse just before an $s I S(a)$ and $600 \mathrm{msec}(b)$ and $3 \mathrm{sec}$ (c) after the $s I S$ are superimposed. The possible activation of a membrane conductance during the late inter-sIS period was further evaluated by analyzing the tonic firing induced by membrane potential depolarization (Fig. 3). The activation of the conductance associated with the post-sIS GABAb inhibitory potential shunted the tonic firing evoked by membrane depolarization to $-57 \mathrm{mV}$ (Fig. $3 A$ ). The pre-sIS firing frequency was completely reestablished within $2 \mathrm{sec}$ from the $s I S$ onset $(n=4)$. The graph in Figure $3 B$ shows the same effect in a different neuron. The return of firing rate to control values after the $s I S$ is illustrated for two different levels of depolarization $(-40$ and $-53 \mathrm{mV})$. These results suggest that no significative changes in membrane conductance correlate to the late part of the inter-sIS potential.

The possibility that the slow inter-sIS potential could be attributable to distal voltage-dependent dendritic conductances activated by the sustained $s I S$-related bursting was evaluated by hindering bursting activity either by passively diffusing the sodium channel blocker QX-314 (80 mM) through the intracellular electrode (Fig. $4 A ; n=8$ ) or by hyperpolarizing membrane potential via steady current injection (Fig. $4 B ; n=3$ ). In both types of experiments the slow depolarizing potential was not abolished.

Finally, because slow depolarizing potentials dependent on nonionotropic glutamate receptors have been demonstrated in cortical neurons (Bianchi and Wong, 1995), the effect of the metabotropic receptor antagonist MCPG applied either by arterial perfusion $(2 \mathrm{~mm} ; n=2)$ or locally in the tissue $(20 \mathrm{~mm} ; n=$ 2 ) was tested. The drug did not affect the $s I S$ periodicity (data not shown).

These results suggest that the inter-sIS period is not associated with the activation of an intrinsic or synaptic membrane conductance. As a possible alternative, modifications of ion concentrations in the extracellular space could lead to inhibition during the silent period. Because transmembrane movement of $\mathrm{K}^{+}$and protons is known to follow massive and synchronous bursting activity (Moody et al., 1974; Heinemann et al., 1977; Somjen, 1984; Jarolimek et al., 1989) (for review, see Jefferys, 1995), the modifications of $\mathrm{K}^{+}$concentration and $\mathrm{pH}$ in the extracellular space were measured during periodic sISs with ion-selective electrodes. The $S I S$ recorded extracellularly consistently correlated to a rapid increase in extracellular $\mathrm{K}^{+}$concentration (peak concentration changes of $0.5-1 \mathrm{~mm}$ from a baseline of $3.5 \mathrm{~mm} ; n=4$; Fig. $5)$. The recovery to control values coincided with the activation of the next $s I S$ s.

Similarly, a fast-rising extracellular alkaline shift (ranging between 0.05 and $0.08 \mathrm{pH}$ units from a baseline of $7.3 ; n=5$ ) that completely decayed before the activation of the next $s I S$ was observed during the inter-sIS period (Fig. 6A). High-frequency afferent stimulation that mimics bursting activity has been shown to induce fast extracellular alkaline transients with rise times in the order of the tens of milliseconds (Gottfried and Chesler, 1996), compatible with the rapid changes we observed. The periodicity of the $s I S$ s (Fig. 6B, top two traces) was disrupted when a tetanic stimulation was applied to the lateral olfactory tract (Fig. $6 \mathrm{~B}$, bottom two traces). High-frequency stimulation also induced a large extracellular $\mathrm{K}^{+}$shift that returned to control values with a time course similar to that of $\mathrm{pH}$ changes (data not shown). The discontinuation of the periodicity correlated to a very large alkaline shift, and the reappearance of the $s I S$ s coincided with the return of $\mathrm{pH}$ to control values (Fig. $6 B$ ). Both increase in $\mathrm{K}^{+}$and alkalinization of the extracellular environment are known to increase neuronal excitability by several means (Rutecki et al., 1985; Church and McLennan, 1989; Chamberlin et al., 1990; Gottfried and Chesler, 1994; Tombaugh and Somjen 1996; Deitmer and Rose, 1996). Because increases in $\mathrm{K}^{+}$and alkalinization occur after a single $s I S$, a gradual transition toward the development of an ictal event would be expected if excitability were enhanced. On the contrary, in our experimental conditions the probability of occurrence of an $S I S$ is reduced during the inter-sIS period, as demonstrated by the inability to generate $s I S$ s from subthreshold potentials in a condition of increased $\mathrm{K}^{+}$illustrated in Figure 5 (top trace, arrowheads).

The observed extracellular alkalinization could be attributable to a rapid transmembrane movement of protons into the neurons associated with the massive bursting and the secondary recurrent synaptic excitation during the $s I S$ (Kraig et al., 1983; Chen and Chesler, 1992; Hartley and Dubinsky, 1993; Deitmer and Rose, 


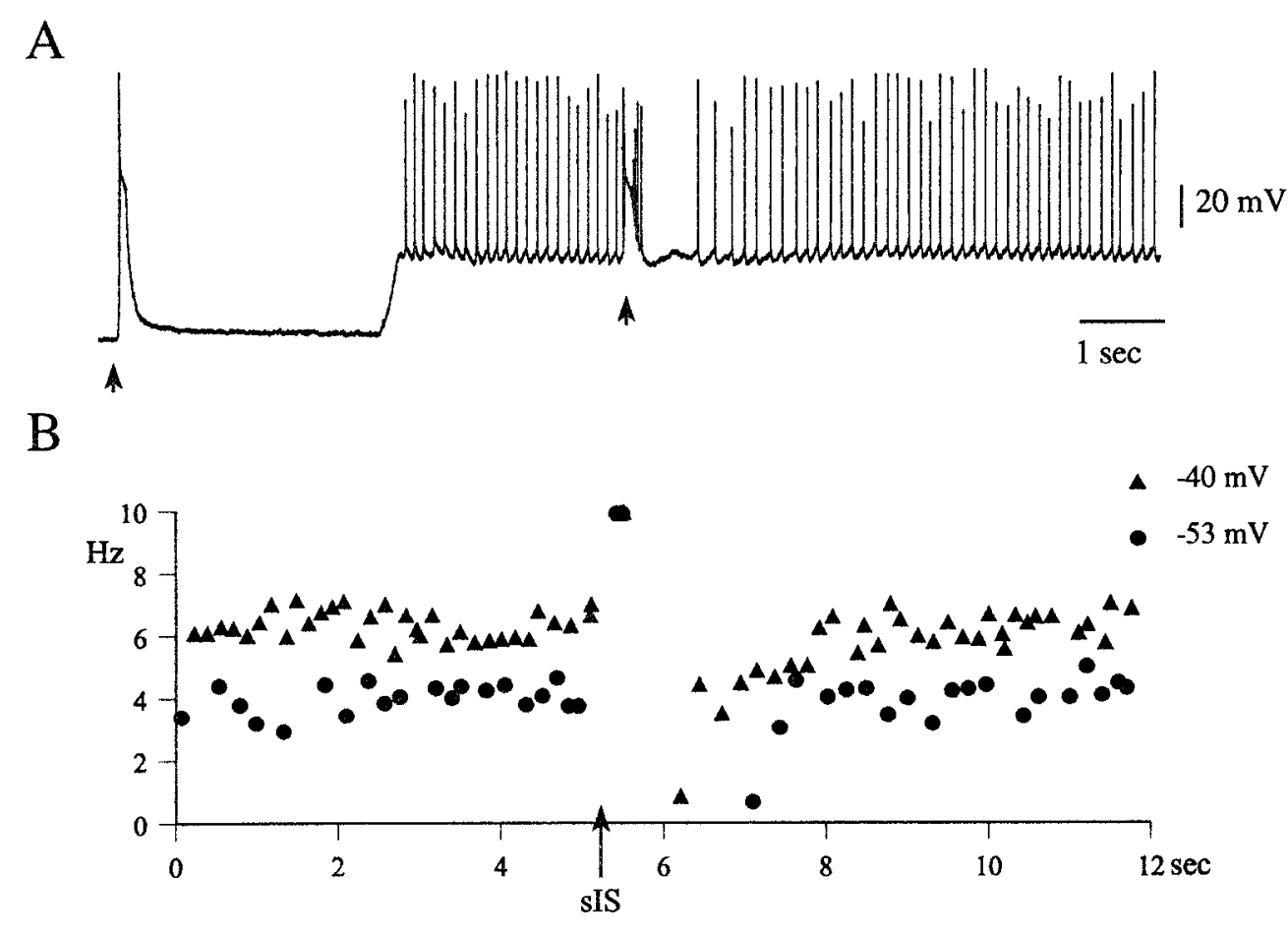
membrane depolarization is transiently interrupted by a $s I S$ ( $A$, arrowheads; resting membrane potential, $-78 \mathrm{mV}$ ). The bottom trace in $A$ shows the intracellular current injection. The graph in $B$ illustrates the changes in firing frequency at two different membrane polarization $(\boldsymbol{\Lambda},-40 \mathrm{mV} ; \boldsymbol{\bullet},-53 \mathrm{mV})$ in another neuron. The tonic firing returned to pre-sIS frequency within $2 \mathrm{sec}$ after an sIS (abscissa, arrow).
Figure 3. The tonic firing evoked by

1996). Intracellular acidification is known to decrease gap junctional conductance (Spray et al., 1981; Perez-Velasquez et al., 1994). If the intracellular acidification determined inter-sIS inhibition via a blockade of the nonsynaptic transfer of excitation among PC neurons, then reducing acidification should cause an increase in the frequency of $s I S \mathrm{~s}$ and should promote a transition toward an ictal discharge. Ammonium chloride $\left(\mathrm{NH}_{4} \mathrm{Cl}\right)$, a compound known to induce transient intracellular alkalinization followed by acidification (Giaume and Korn, 1982; Thomas, 1984; Perez-Velasquez et al., 1994), was used to test this hypothesis. $\mathrm{NH}_{4} \mathrm{Cl}$ applied via the arterial system $(10-20 \mathrm{~mm} ; n=6)$ induced a gradual increase in excitability followed by a prolonged inhibition (up to $1 \mathrm{hr}$ ) on $\mathrm{NH}_{4} \mathrm{Cl}$ washout. The extracellular recordings illustrated in Figure $7 A$ show that the transient increase in excitability is characterized by the activation of spontaneous, longlasting (1-2 sec) afterdischarges (after 2-5 min of perfusion) followed by a disappearance of the $s I S$ (after 5-10 min of perfusion). The polysynaptic component of the field responses evoked by low-intensity LOT stimulation, but not the monosynaptic response, was reduced by $\mathrm{NH}_{4} \mathrm{Cl}$, suggesting that the intrinsic excitability of $\mathrm{PC}$ neurons was not increased by $\mathrm{NH}_{4} \mathrm{Cl}$ (data not shown). When intracellular recordings were performed, no changes in input membrane resistance were observed during $\mathrm{NH}_{4} \mathrm{Cl}$ perfusion $(n=2$; data not shown). The effects of $\mathrm{NH}_{4} \mathrm{Cl}$ were reverted within $40-60$ min after washout. In two of six tests prolonged ictal discharges were observed during $\mathrm{NH}_{4} \mathrm{Cl}$ perfusion (Fig. 7B, middle traces). The LOT stimulation-evoked activity was preserved during the silent period (Fig. 7B, middle trace, arrowhead), suggesting that the $\mathrm{NH}_{4} \mathrm{Cl}$-induced inhibition was not coupled to a spreading depression-like phenomenon.

Because a decrease in electrotonic coupling between neurons could substantially reduce excitability, we tested the possibility that a reduction of gap junction function could prevent further

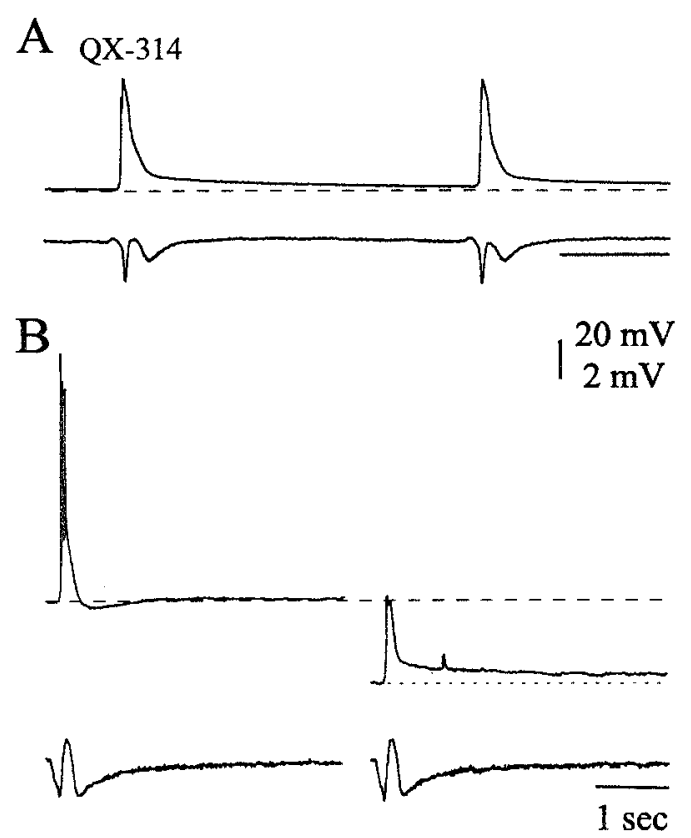

Figure 4. The depolarization during the inter-sIS period is not dependent on the bursting activity associated with the $s I S$. $A$, Bursting activity was blocked by applying intracellularly the sodium channel blocker QX-314 (80 mM). The frequency of the $s I S \mathrm{~s}$ was not affected by the drug (resting membrane potential, $-76 \mathrm{mV}$ ). QX-314 also abolished the GABAb response (de Curtis et al., 1998). The top and bottom traces represent the simultaneous intracellular and extracellular recordings performed $1 \mathrm{hr}$ after transient bicuculline ejection. $B$, In a different neuron the bursting activity was abolished by artificially hyperpolarizing the cell with intracellular current injection. The inter$s I S$ depolarization was not affected. Because of the depolarized resting membrane potential $(-72 \mathrm{mV}$, dotted line $)$ in this cell, the slow depolarization was not prominent in the resting condition but become evident when the membrane potential was hyperpolarized. 
FP
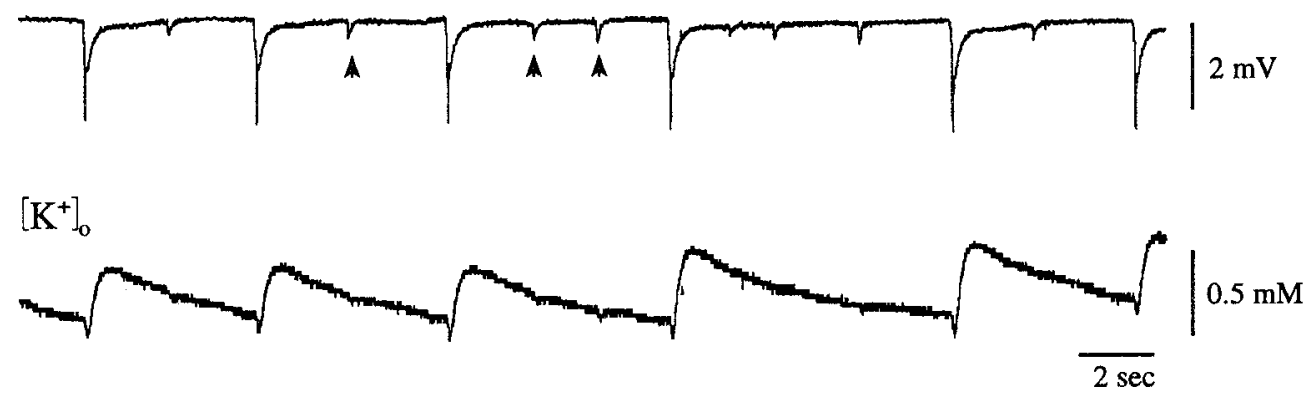

Figure 5. The inter-sIS period is coupled to an increase in extracellular $\mathrm{K}^{+}$ concentration. Simultaneous extracellular field potential (FP, top traces) and potassium ion $\left(\left[\mathrm{K}^{++}\right]_{o}\right.$, bottom traces $)$ recordings are shown. Each $s I S$ was associated with a rapid increase in $\mathrm{K}^{+}$ concentration, which returned to control values before the activation of the next sIS. Note that spontaneous field potentials are below the threshold for a population spike (top trace, arrows) were observed even when the concentration of extracellular $\mathrm{K}^{+}$was higher then control values just before an $s I S$.
sIS to be generated. The gap junctional blocker octanol (Peinado et al., 1993) applied either by arterial perfusion ( $0.2 \mathrm{~mm} ; n=5)$ or locally in the cortex $(2 \mathrm{~mm} ; n=2)$ induced a complete and reversible abolition of the $S I S$ (Fig. $8 A$ ). Because octanol has been shown to decrease T-type calcium conductances (Scott et al., 1990), we tested the effect of the T-type calcium condutance blocker nickel to exclude the involvement of highthreshold calcium spike modulation in the abolition of $s I S \mathrm{~s}$. Local application of nickel $(50 \mu \mathrm{M} ; n=2)$ did not modify $s I S$ periodicity (data not shown). Arterial perfusion with another gap junction blocker, $18 \alpha$-glycyrrhetinic acid (20 $\mu \mathrm{M}$; Davidson et al., 1986; Blanc et al., 1998), reduced the population spike component of the $s I S$ and progressively decreased their periodicity until a quasi-complete abolition was obtained after 5 min (Fig. $8 B ; n=3$ ). In two experiments the effect was reverted after $5-10 \mathrm{~min}$ of washout.

\section{DISCUSSION}

The study demonstrates that spontaneous interictal potentials induced by transient application of bicuculline in the piriform cortex of the isolated guinea pig brain recur periodically. Two possible conditions may account for the inter-sIS silent period: (1) the hyperexcitable cortical network requires some time to build up and to reach threshold for the activation of the next spontaneous synchronous event after the activation of a $S I S$; or (2) the cortex is transiently inhibited after an $S I S$ and cannot be reexcited until such inhibition is removed.

\section{A}

FP<smiles>CCCI</smiles>
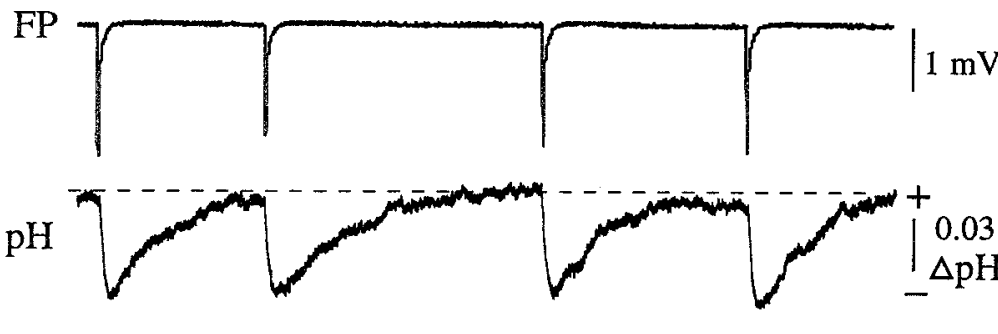

$5 \mathrm{sec}$

B
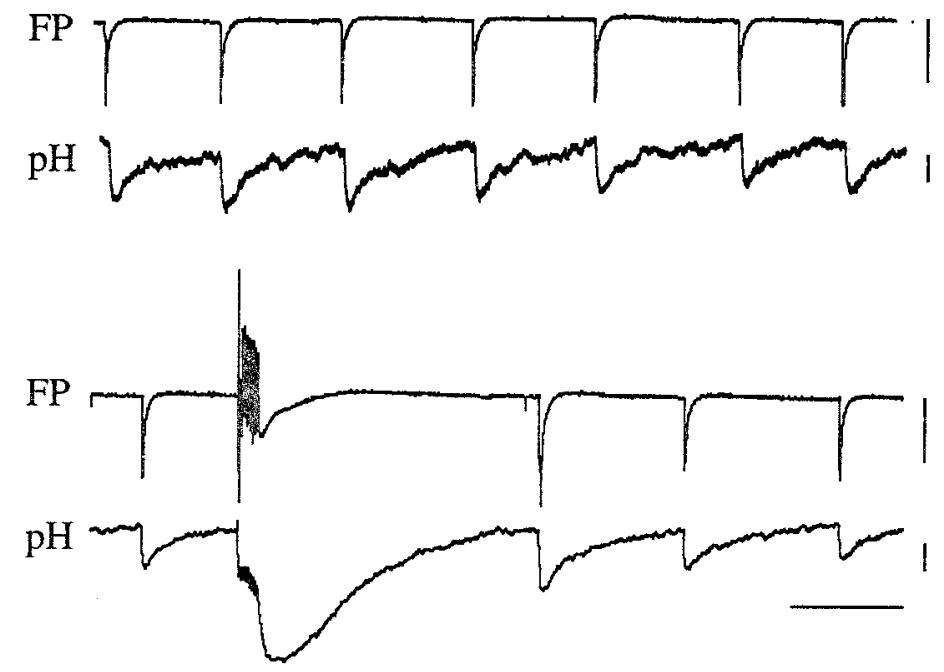

Figure 6. The inter-sIS period correlated to a fast-rising alkalinization of the extracellular space. As for Figure 5, simultaneous recordings of the field potential ( $F P$, top traces) and the extracellular $\mathrm{pH}$ (bottom traces) are illustrated. $A$, The fast $\mathrm{pH}$ transient returned to control values before the activation of a subsequent $s I S$. B. The periodicity of the sIS (top two traces) was discontinued by the large $\mathrm{pH}$ shift induced by LOT tetanic stimulation $(10 \mathrm{~Hz}, 1 \mathrm{sec})$ that massively activated the piriform cortex (bottom two traces). Periodic $s I S$ were restored when the alkaline shift recovered. 
A

Figure 7. $\mathrm{NH}_{4} \mathrm{Cl}$ induces an increase in excitability, followed by a decrease in excitation on washout. $A$, The top trace shows periodic $s I S \mathrm{~s}$ in the control condition. Within 2 min of perfusion with $\mathrm{NH}_{4} \mathrm{Cl}(20 \mathrm{~mm}, \mathrm{pH} 7.3)$ afterdischarges are generated (second trace from top) and are followed at $10 \mathrm{~min}$ by a silent period (third trace). In this experiment the effect of $\mathrm{NH}_{4} \mathrm{Cl}$ was completely reverted after $1 \mathrm{hr}$ of washout (bottom trace). B, In a different experiment, $\mathrm{NH}_{4} \mathrm{Cl}$ disrupted $s I S$ synchronization and determined an increase in their frequency (second trace). After $5 \mathrm{~min}$ of $\mathrm{NH}_{4} \mathrm{Cl}$ perfusion $(20 \mathrm{mM})$ a seizurelike event spontaneously occurred (third trace), followed by a silent period that lasted $40 \mathrm{~min}$ ( fourth trace). The arrowhead points to the field response evoked by LOT during the silent period. An incomplete recovery was obtained in this experiment (as shown in the bottom trace, after $\mathrm{NH}_{4} \mathrm{Cl}$ perfusion $s I S \mathrm{~s}$ were not as large and synchronous as in control conditions).

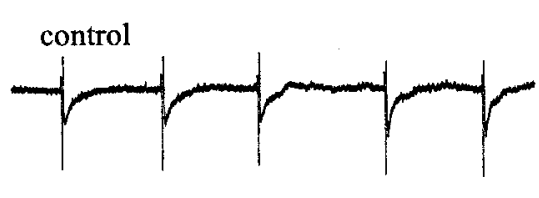

$\mathrm{NH}_{4} \mathrm{Cl} 10 \mathrm{~min}$

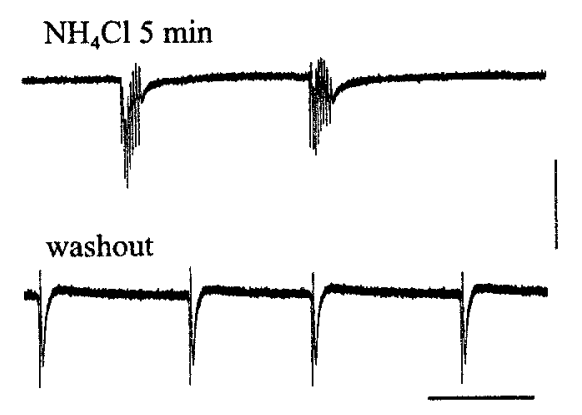

B

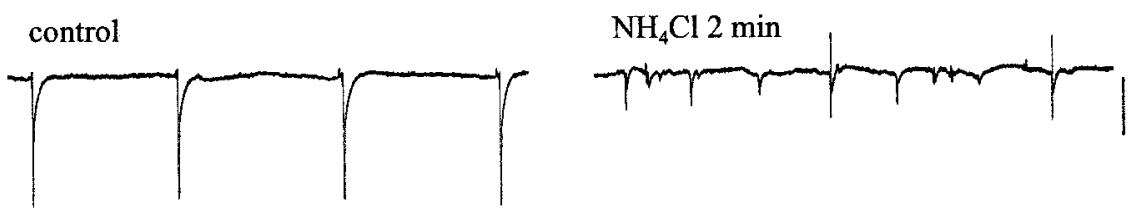

Our experiments demonstrate that a single $s I S$ induces extracellular ionic shifts that would be expected to produce excitation but paradoxically are associated with a predominant inhibition. Moody et al. (1974) demonstrated that $\mathrm{K}^{+}$elevation is maximal $150-400 \mathrm{msec}$ after an interictal population burst and slowly decays with a time course of several seconds. Similarly, in our experiments the extracellular $\mathrm{K}^{+}$concentration was maximally increased just after an $S I S$ and remained elevated for $5-10 \mathrm{sec}$. High extracellular $\mathrm{K}^{+}$is known to slow down repolarization of presynaptic terminals and to increase the presynaptic release of neurotransmitter, therefore augmenting the duration and frequency of spontaneous EPSPs (Chamberlin et al., 1990; Traub and Dingledine, 1990). The probability of activating spontaneous EPSPs in our model should be maximal $\sim 2 \mathrm{sec}$ after the preceding $s I S$ discharge, when recurrent GABAb synaptic inhibition ceases and extracellular $\mathrm{K}^{+}$is still elevated (Traub and Dingledine, 1990). As a consequence, if the buildup of synaptic excitation would be the only mechanism for $s I S$ generation, $s I S$ s should be facilitated at the end of post-sIS GABAb-mediated inhibitory potential. This is not the case, because $s I S \mathrm{~s}$ are reactivated after a much longer period $(5-10 \mathrm{sec})$ in our experiments.

A condition of enhanced excitability would also be expected to result from the extracellular alkalinization induced by the synchronous neuronal firing associated with the $s I S$ (for review, see Chesler and Kaila, 1992; Deitmer and Rose, 1996).
Figure 8. Drugs that reduce gap junction function abolished the $s I S \mathrm{~s}$. $A$, Octanol $(20 \mathrm{~mm})$ applied by arterial diffusion for 2 min transiently blocked the $s I S$ s. During octanol small-amplitude potentials below the threshold for $s I S$ occurred sporadically (arrow). The presence of an extracellular correlate suggests that such subthreshold potentials are population events. When the effect of octanol was washed out, a slower periodicity of $s I S$ was observed in most of the experiments. Resting membrane potential, $-73 \mathrm{mV}$. $B, 18 \alpha-$ Glycyrrhetinic acid $(20 \mu \mathrm{M})$ applied by arterial perfusion also reversibly abolished $s I S$ s. The effect was associated with a reduction in the amplitude of the $s I S$ population spikes.

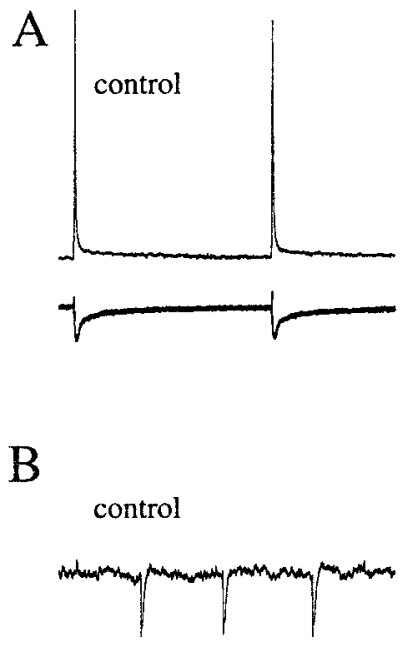

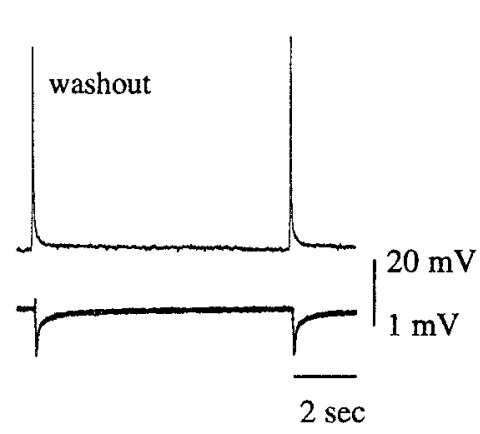

glycyrrethinic ac.
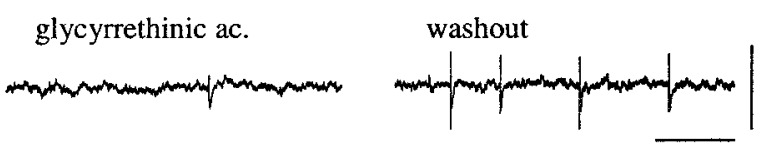
Several studies demonstrated that extracellular alkalinization enhances $\mathrm{Na}^{+}$and $\mathrm{Ca}^{+2}$ voltage-activated conductances in hippocampal neurons (Tombaugh and Somjen, 1996), increases NMDA receptor-dependent activity (Tang et al., 1990; Traynelis and Cull-Candy, 1990; Gottfried and Chesler, 1994), and promotes seizure activity (Jarolimek et al., 1989). Thus, like the increases in extracellular $\mathrm{K}^{+}$, the post-sIS alkalinization produces a condition in the extracellular microenvironment that would favor excitation, in striking contrast with the observation of a silent inter-sIS period. The absence of such an increase in excitability is demonstrated by the observation that spontaneous potentials below the threshold for an SIS (Fig. 5, arrows) do not evoke a population spike during the silent period, despite the concurrent elevations in extracellular $\mathrm{K}^{+}$ and $\mathrm{pH}$. Indeed, a transient decrease in excitability occurs during these ionic shifts induced by the activation of $s I S$.

A transient inhibition during the silent period could be attributable either to activation of a shunting conductance or to reduced coupling between neurons. As mentioned before, the early phase of the inter-sIS potential correlates to a GABAb receptor-mediated inhibitory synaptic potential (de Curtis et al., 1998). The late component of the inter-sIS period is paralleled by a slowly decaying depolarization that is (1) abolished at membrane potentials positive to $-35 \mathrm{mV}$, (2) not coupled to a reduction in membrane resistance (Fig. 2), and (3) not dependent on the intracellular activation of the $S I S$ burst. These observations suggest that the slow inter-sIS depolarization is not mediated by the activation of an established synaptic or intrinsic membrane conductance. Bal and McCormick (1996) showed that the slow depolarizing potentials responsible for the interspindle silent period are mediated by the persistent activation of a hyperpolarization-activated cation current in thalamic neurons. The possible role of such a current can be excluded in our experimental conditions, because the selective intracellular blocker of the hyperpolarization activated conductance, QX-314 (Perkins and Wong, 1995), does not abolish the inter-sIS slow depolarization (Fig. $4 A$ ). In cortical neurons metabotropic glutamate receptors have been shown to sustain slow depolarizing events (Bianchi and Wong, 1995) that might presynaptically depress synaptic transmission (Burke and Hablitz, 1994) and could be responsible for the inter-sIS silent period. This inhibitory mechanism is ruled out in our model by the demonstration that the metabotropic glutamate receptor MCPG does not alter sIS periodicity. We concluded that none of the membrane conductances known to reduce excitability in cortical neurons is activated during the late depolarizing potential associated with the inter-sIS period.

We noticed that the return to resting membrane potential values of the slow post-sIS depolarization paralleled the slow, monotonic decay of $\mathrm{K}^{+}$concentration measured with ionselective electrodes during the silent period. This observation suggest that the post-sIS membrane depolarization could be attributable to a modification of the $\mathrm{K}^{+}$equilibrium potential consequent to the transient elevation of the extracellular $\mathrm{K}^{+}$ concentration associated with the population sIS discharge (Whisler and Johnston, 1978; Rutecki et al., 1985; Haglund and Schwartzkroin, 1990; Otis et al., 1993). The post-sIS depolarization had an amplitude of 4-6 $\mathrm{mV}$ just after the spike component of the sIS recorded intracellularly with QX-314, a condition that blocks the GABAb-mediated IPSP and reveals the slow depolarization. Assuming that (1) at resting membrane potential values (approximately $-80 \mathrm{mV}$ ) the membrane of piriform cortex neurons is permeable mainly to $\mathrm{K}^{+}$, (2) the $\left[\mathrm{K}^{+}\right]_{\mathrm{i}}$ measured at $32^{\circ} \mathrm{C}$ for a $-87 \mathrm{mV} \mathrm{K}^{+}$equilibrium potential (Otis et al., 1993) is $100 \mathrm{~mm}$, and (3) the experimental $\left[\mathrm{K}^{+}\right]_{\mathrm{o}}$ is $3.5 \mathrm{~mm}$, the $0.5-1 \mathrm{~mm}$ increase in $\left[\mathrm{K}^{+}\right]_{\mathrm{o}}$ measured in our experiments just after the $s I S$ determined according to the Nernst-Planck equation a $3.5-6.5 \mathrm{mV}$ shift in the $\mathrm{K}^{+}$equilibrium potential, compatible with the amplitude of the observed depolarization.

The extracellular alkalinization associated with neuronal activity is supposed to be caused by a rapid intracellular acidification mediated by transmembrane flow of protons from the extracellular space into neurons (Kraig et al., 1983) either through ion channels activated by excitatory and inhibitory neurotransmitters or by calcium-proton exchange (Chen and Chesler, 1992; Smith et al., 1994) (for review, see Chesler and Kaila, 1992; Deitmer and Rose, 1996). $\mathrm{NH}_{4} \mathrm{Cl}$ has been applied in our experiments to test whether by preventing intracellular acidification we could increase excitability and interfere with the mechanisms that generate the silent inter-sIS period. $\mathrm{NH}_{4} \mathrm{Cl}$ is known to enter cells via $\mathrm{K}^{+}$channels (Moser, 1987) and to induce a transient intracellular alkalinization followed by intracellular acidification on washout (Giaume and Korn, 1982; Thomas, 1984; Perez-Velasques et al., 1994). The results show that the intracellular alkalinization presumably induced during the early phases of $\mathrm{NH}_{4} \mathrm{Cl}$ perfusion promotes a fast transition toward ictal-like discharges (Fig. 7A,B). Moreover, during the $\mathrm{NH}_{4} \mathrm{Cl}$ washout, when intracellular acidification is expected to occur, $s I S$ s are completely abolished. These observations strongly suggest that (1) a marked enhancement in neuronal excitability is produced by contrasting $s I S$-induced intracellular acidification, and (2) intracellular acidification promotes a condition of low excitability during which no sIS can be generated. The possibility that the transitory enhancement in excitability we observed could be attributable to $\mathrm{NH}_{4} \mathrm{Cl}$-induced increase of calcium conductances, as demonstrated in chick sensory neurons (Mironov and Lux, 1993), can be excluded because (1) membrane input resistance did not change during $\mathrm{NH}_{4} \mathrm{Cl}$ perfusion, and (2) the polysynaptic component of the field responses evoked by low-intensity LOT stimulation below the threshold for the activation of interictal epileptiform discharge, but not the monosynaptic response, was reduced by $\mathrm{NH}_{4} \mathrm{Cl}$.

Both intracellular acidification or extracellular alkalinization have been shown to influence dye coupling (Church and Baimbridge, 1991) and bursting behavior of neurons (Church and McLennan, 1989; Valiante et al., 1996) by reducing gap junction function (Spray et al., 1981) and consequently by decreasing synchronization of neuronal firing (Perez-Velazquez, 1994). This mechanism could hypothetically account for the transient decrease in intrinsic neuronal excitability during the inter-sIS period. Nonsynaptic intercellular diffusion of action potentials generated during the $s I S$ might be reduced by transient gap junction impairment so that population excitability decreases and repetitive activation of the neurons that just generated a $S I S$ is prevented. The abolition of $s I S \mathrm{~s}$ induced by application of gap junction blockers octanol and $18 \alpha$-glycyrrhetinic acid supports this hypothesis.

The results shown here suggest that changes in $\mathrm{pH}$ should not be considered an inconsequential epiphenomenon generated during interictal spiking but, rather, may be a primary factor that regulates neuronal excitability and controls epileptiform discharges. Patterns of recurrent interictal activity such as periodic sharp waves or spikes similar to those described in this study have been described in experimental models of epileptogenesis and are commonly observed as interictal elec- 
troencephalographic abnormalities in epileptic patients suffering of idiopathic or postlesional partial epilepsy (Chatrian et al., 1964; Beaussat et al., 1972). Even if the basic alterations expressed in different epileptic conditions are highly heterogeneous, common mechanisms similar to those described in the present paper could be postulated for the generation of periodic epileptiform events, and their functional implications can be exploited to understand the process of epileptogenesis.

\section{REFERENCES}

Bal T, McCormick DA (1996) What stops synchronized thalamocortical oscillations? Neuron 17:297-308.

Beaussat M (1972) Benign epilepsy of children with Rolandic centrotemporal paroxysmal foci. A clinical entity. Study of 221 cases. Epilepsia 11:795-811.

Bianchi R, Wong RKS (1995) Excitatory synaptic potentials dependent on metabotropic glutamate receptor activation in guinea pig hippocampal pyramidal cells. J Physiol (Lond) 487:663-676.

Biella G, de Curtis M (1995) Associative potentials in the PC of the in vitro isolated guinea pig brain. Eur J Neurosci 7:54-64.

Blanc EM, Bruce-Keller AJ, Mattson MP (1998) Astrocytic gap junctional communication decreases neuronal vulnerability to oxidative stress-induced disruption of calcium homeostasis and cell death. J Neurochem 70:958-970.

Burke JP, Hablitz JJ (1994) Presynaptic depression of synaptic transmission mediated by activation of metabotropic glutamate receptors in rat neocortex. J Neurosci 14:5120-5130.

Chamberlin NL, Traub RD, Dingledine R (1990) Role od EPSPs in initiation of spontaneous synchronized burst firing in rat hippocampal neurons bathed in high potassium. J Neurophysiol 64:1000-1008.

Chatrian GE, Shaw CM, Leffmann H (1964) The significance of periodic lateralized epileptiform discharges in EEG: an electrographic, clinical and pathological study. Electroencephalogr Clin Neurophysiol 17:177-193.

Chen CT, Chesler M (1992) Extracellular alkaline shifts in rat hippocampal slices are mediated by NMDA and non-NMDA receptors. J Neurophysiol 68:342-344.

Chesler M, Kaila K (1992) Modulation of $\mathrm{pH}$ by neuronal activity. Trends Neurosci 15:396-402.

Church J, Baimbridge KG (1991) Exposure to high-pH medium increases the incidence and extent of dye coupling between rat hippocampal CA1 pyramidal neurons in vitro. J Neurosci 11:3289-3295.

Church J, McLennan H (1989) Electrophysiological properties of rat CA1 pyramidal neurons in vitro modified by changes in extracellular bicarbonate. J Physiol (Lond) 415:85-108.

Davidson JS, Baumgarten IM, Harley EH (1986) Reversible inhibition of intercellular junctional communication by glycyrrhetinic acid. Biochem Biophys Res Commun 134:29-36.

de Curtis M, Parè D, Llinas R (1991) The electrophysiology of the olfactory-hippocampal circuit in the isolated and perfused adult mammalian brain in vitro. Hippocampus 1:341-354.

de Curtis M, Biella G, Forti M, Panzica F (1994) Multifocal epileptiform activity induced by restricted convulsing ejections in the piriform cortex of the isolated guinea pig brain. J Neurophysiol 71:2463-2476.

de Curtis M, Radici C, Forti M (1998) Cellular mechanisms underlying spontaneous interictal spikes in a model of focal cortical epileptogenesis. Neuroscience, in press.

Deitmer JW, Rose CR (1996) pH regulation and proton signaling by glia cells. Prog Neurobiol 48:73-103.

Forti M, Biella G, Caccia S, de Curtis M (1997) Persistent excitability changes in the piriform cortex of the isolated guinea pig brain after transient exposure to bicuculline. Eur J Neurosci 9:435-451.

Giaume C, Korn H (1982) Ammonium sulfate induced uncoupling of crayfish axons with and without increased junctional conductance. Neuroscience 7:1723-1730.

Gottfried JA, Chesler M (1994) Endogenous H+ modulation of NMDA receptor-mediated EPSCs revealed by carbonic anhydrase inhibition in rat hippocampus. J Physiol (Lond) 478:373-378.

Gottfried JA, Chesler M (1996) Temporal resolution of activity- dependent $\mathrm{pH}$ shifts in rat hippocampal slices. J Neurophysiol 76:2804-2807.

Haberly LB, Bower JM (1989) Olfactory cortex: model circuit for study of associative memory?. Trends Neurosci 12:258-264.

Haglund MM, Schwartzkroin PA (1990) Role of Na-K pump potassium regulation and IPSPs in seizures and spreading depression in immature rabbit hippocampal slices. J Neurophysiol 63:225-239.

Hartley Z, Dubinsky JM (1993) Changes in intracellular pH associated with glutamate excitotoxicity. J Neurosci 13:4690-4699.

Heinemann U, Lux HD, Gutnick MJ (1977) Extracellular free calcium and potassium during paroxysmal activity in the cerebral cortex of the rat. Exp Brain Res 27:237-243.

Jarolimek W, Misgeld U, Lux HD (1989) Activity dependent alkaline and acid transients in guinea pig hippocampal slices. Brain Res 505:225-232.

Jefferys JGR (1995) Nonsynaptic modulation of neuronal activity in the brain: electric currents and extracellular ions. Physiol Rev 75:689-723.

Kraig RP, Ferreira-Filho CR, Nicholson C (1983) Alkaline and acid transients in cerebellar microenvironment. J Neurophysiol 49:831-850.

Lebovitz RM (1979) Autorhythmicity of spontaneous interictal spike discharge at hippocampal penicillin focus. Brain Res 172:35-55.

Leung LS (1990) Spontaneous hippocampal interictal spikes following local kindling: time-course and relation to behavioral seizures. Brain Res 513:308-314.

Llinas R, Yarom Y, Sugimori M (1981) Isolated mammalian brain in vitro: new technique for analysis of electrical activity of neuronal circuit function. Fed Proc 40:2240-2245.

Mironov SL, Lux HD (1993) $\mathrm{NH}_{4} \mathrm{Cl}$-induced inward current and cytoplasmic calcium transients in chick sensory neurones. NeuroReport 4:1055-1058.

Moody WJ, Futamachi KJ, Prince DA (1974) Extracellular potassium activity during epileptogenesis. Exp Neurol 42:248-263.

Moser H (1987) Electrophysiological evidence for ammonium as substitute for potassium in activating the sodium pump in crayfish sensory neuron. Can J Physiol Pharmacol 65:141-145.

Muhlethaler M, de Curtis M, Walton K, Llinas R (1993) The isolated and perfused brain of the guinea pig in vitro. Eur $\mathrm{J}$ Neurosci 5:915-926.

Otis TS, De Koninck Y, Mody I (1993) Characterization of synaptically elicited GABAb responses using patch clamp recordings in rat hippocampal slices. J Physiol (Lond) 463:391-407.

Peinado A, Yuste R, Katz LC (1993) Extensive dye coupling between rat neocortical neurons during the period of circuit formation. Neuron 10:103-114

Pelletier MR, Carlen PL (1997) Repeated tetanic stimulation in piriform cortex in vitro: epileptogenesis and pharmacology. J Neurophysiol 75:4069-4079.

Perez-Velasquez JL, Valiante TA, Carlen PL (1994) Modulation of gap junctional mechanisms during $\mathrm{Ca}$-free induced field burst activity, a possible role for electrotonic coupling in epileptogenesis. J Neurosci 14:4308-4317.

Perkins KL, Wong RKS (1995) Intracellular QX-314 blocks the hyperpolarization-activated inward current Iq in hippocampal CA1 pyramidal cells. J Neurophysiol 73:911-915.

Prince DA (1971) Cortical cellular activities during cyclically occurring inter-ictal epileptiform discharges. Electroencephalogr Clin Neurophysiol 31:496-484.

Rutecki PA, Lebeda FJ, Johnston D (1985) Epileptiform activity induced by changes in extracellular potassium in hippocampus. J Neurophysiol 54:1363-1374.

Schneiderman JH, Mac Donald JF (1989) Excitatory amino acid blockers differentially affect bursting of in vitro hippocampal neurons in two pharmacological models of epilepsy. Neuroscience 3:593-603.

Scott RH, Wootton JF, Dolphin AC (1990) Modulation of the T-type calcium currents by photoactivation of intracellular $5^{\prime}-O(3-$ thio)triphosphate. Neuroscience 38:285-294.

Smith SE, Gottfried HJ, Chen JCT, Chesler M (1994) Calcium dependence of glutamate receptor-evoked alkaline shifts in hippocampus. Neuroscience 5:2441-2445.

Somjen GG (1984) Acidification of interstitial fluid in hippocampal formation caused by seizures and by spreading depression. Brain Res 311:186-188.

Spray DC, Harris AL, Bennet MVL (1981) Gap junctional conductance 
is a simple and sensitive function of intracellular $\mathrm{pH}$. Science 211:712-715.

Tang CM, Dichter M, Morad M (1990) Modulation of the NMDA channel by extracellular $\mathrm{H}^{+}$. Proc Natl Acad Sci USA 87:6445-6449.

Thomas RC (1984) Extracellular displacement of intracellular $\mathrm{pH}$ and the mechanisms of its subsequent recovery. J Physiol (Lond) 354:3-22.

Tombaugh GC, Somjen GG (1996) Effects of extracellular pH on voltage gated $\mathrm{Na}^{+}, \mathrm{K}^{+}$and $\mathrm{Ca}^{+2}$ currents in isolated rat CA1 neurons. J Physiol (Lond) 493:719-732.

Traub RD, Dingledine R (1990) Model of synchronized epileptiform bursts induced by high potassium in CA3 region of rat hippocampal slice: role of spontaneous EPSPs in initiation. J Neurophysiol 64:1009-1018.
Traub RD, Wong RKS (1982) Cellular mechanisms of neuronal synchronization in epilepsy. Science 216:745-747.

Traub RD, Miles R, Jefferys JGR (1993) Synaptic and intrinsic conductances shape picrotoxin-induced synchronizd after-discharges in the guinea pig hippocampal slice. J Physiol (Lond) 461:525-547.

Traynelis SF, Cull-Candy SG (1990) Proton inhibition of NMDA receptors in cerebellar neurons. Nature 345:347-350.

Valiante TA, Perez Velasquez JL, Jahromi SS, Carlen PL (1996) Coupling potentials in CA1 neurons during calcium-free-induced field burst activity. J Neurosci 15:6946-6956.

Whisler JW, Johnston D (1978) Epileptogenesis: a model for the involvement of slow membrane events and extracellular potassium. J Theor Biol 75:271-288. 